\title{
Les môles hydatiformes partielles au Maroc : étude épidémiologique et clinique
}

\author{
H. Boufettal, ${ }^{1}$ P. Coullin, ${ }^{2}$ S. Mahdaoui, ${ }^{1}$ M. Noun, ${ }^{1}$ S. Hermas ${ }^{7}$ et N. Samouh ${ }^{7}$
}

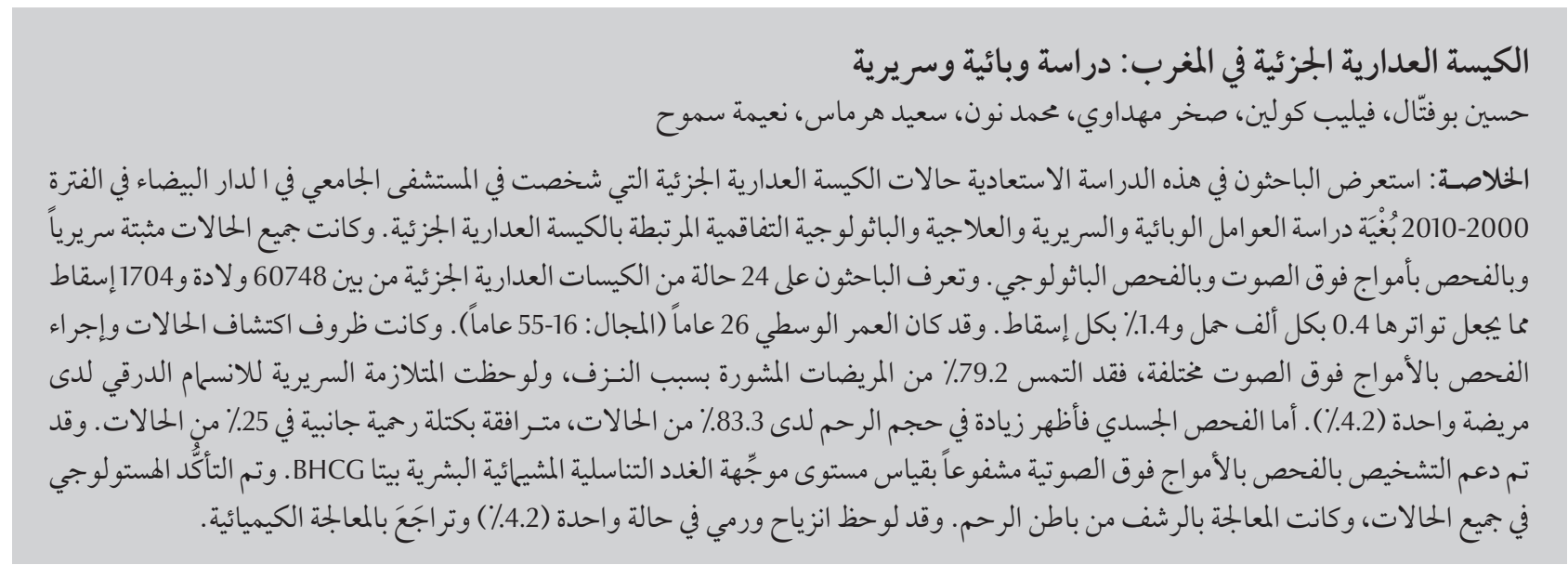

RÉSUMÉ La présente étude rétrospective a examiné les cas de môle hydatiforme partielle (MHP) diagnostiqués au CHU de Casablanca de 2000 à 2010 et rapporte le profil épidémiologique, clinique, thérapeutique et évolutif de cette entité pathologique. Ont été inclus tous les cas de MHP évoqués échographiquement et cliniquement puis confirmés à l'examen anatomo-pathologique. Nous avons recensé 24 cas de MHP parmi 60748 accouchements et 1704 avortements spontanés, ce qui donne une fréquence de 0,4 pour 1000 grossesses et 1,4 \% des avortements. La moyenne d'âge était de 26 ans (extrêmes 16-55 ans). Les circonstances de découverte clinique et échographique étaient variées : 79,2 \% des patientes consultaient pour des métrorragies ; un syndrome thyrotoxicosique clinique était retrouvé chez une patiente (4,2\%). L'examen physique montrait un utérus augmenté de taille dans 83,3 \% des cas associé à une masse latéro-utérine dans 25 \% des cas. Le diagnostic était étayé par un examen échographique associé à un dosage de $\beta$-HCG plasmatique. La confirmation était histologique dans tous les cas et le traitement était une aspiration endo-utérine. La dérive néoplasique a été observée dans un cas $(4,2 \%)$ qui a évolué vers la rémission sous chimiothérapie.

\section{Partial hydatidiform mole in Morocco: an epidemiological and clinical study}

ABSTRACT This retrospective study reviewed cases of partial hydatidiform mole (PHM) diagnosed at the University Hospital in Casablanca from 2000 to 2010 in order to examine the epidemiological, clinical, therapeutic and progressive pathological factors associated with PHM. All PHM cases confirmed clinically and sonographically at pathological examination were included. We identified 24 cases of PHM among 60748 births and 1704 abortions, giving a frequency of 0.4 per 1000 pregnancies and $1.4 \%$ of abortions. The mean age was 26 years (range: 16-55 years). The circumstances of discovery and clinical ultrasound varied: $79.2 \%$ of patients sought consultation for bleeding; clinical thyrotoxicosis syndrome was found in 1 patient (4.2\%). Physical examination showed increased uterine size in $83.3 \%$ of cases associated with a latero-uterine mass in $25 \%$. The diagnosis was supported by an ultrasound examination combined with measurement of plasma $\beta$ HCG. Histological confirmation was made in all cases and treatment was endo-uterine aspiration. Neoplastic drift was observed in 1 case $(4.2 \%)$ which went into remission with chemotherapy.

${ }^{7}$ Service de gynécologie-obstétrique "C ", Faculté de Médecine et de Pharmacie, Centre hospitalier universitaire Ibn Rochd, Centre d'Études Doctorales de Biologie Moléculaire et Génétique, Faculté de Médecine et de Pharmacie, Université Hassan II - Aïn Chock, Casablanca (Maroc) (Correspondance à adresserà H. Boufettal : mohcineb@yahoo.fr).

2Institut national de la santé et de la recherche médicale (INSERM), Unité 782, Endocrinologie et génétique de la reproduction et du développement, Université Paris-Sud, Clamart (France).

Reçu : 10/01/12; accepté : 12/02/12 


\section{Introduction}

La maladie trophoblastique gestationnelle (MTG) correspond à une transformation kystique des villosités choriales associée à une prolifération du trophoblaste avec sécrétion excessive de l'hormone choriogonadotrophine [1]. Les môles hydatiformes complètes (MHC) et partielles (MHP) sont des entités bénignes. Elles peuvent évoluer, avec des probabilités différentes, vers des entités cliniquement malignes appelées tumeurs trophoblastiques gestationnelles (TTG) $[1,2]$.

La MHP est généralement une triploïdie diandrique. Elle dérive de la fécondation d'un ovule normal par deux spermatozoïdes (dispermie) ou un spermatozoïde dupliqué, demeuré diploïde [3]. Elle est caractérisée par une hyperplasie focalisée et discrète du trophoblaste, une dégénérescence localisée des villosités choriales, une structure fotale ou un tissu embryonnaire identifiable $[1,4]$.

Les MHP et MHC présentent des caractéristiques histopathologiques et cytogénétiques ainsi qu'une évolution très distinctes. L'étude de la MHP et de la MHC séparément montre une différence importante en ce qui concerne l'association de facteurs, notamment l'âge maternel, la parité, le groupe sanguin, le rôle des facteurs exogènes, cette association étant plus fréquente dans l'une que dans l'autre $[1,2]$.

Nous rapportons une série de môles hydatiformes partielles, à travers laquelle nous allons présenter nos résultats épidémiologiques, cliniques, thérapeutiques et évolutifs et distinguer les particularités de cette entité.

\section{Méthodes}

Cette étude rétrospective des MHP marocaines porte sur une période de 11 années, qui s'étale de janvier 2000 à décembre 2010. Elle a été réalisée sur la base de l'activité du service de gynécologie-obstétrique « $\mathrm{C} \gg \mathrm{du}$ Centre Hospitalier Universitaire Ibn Rochd de Casablanca au Maroc, où 24 cas de MHP ont ainsi pu être colligés.

Les critères d'inclusion étaient les suivants : toutes les MHP évoquées échographiquement et cliniquement puis confirmées à l'examen anatomo-pathologique. Nous avons étudié en particulier les données épidémiologiques, cliniques, thérapeutiques et évolutives des MHP. Le test du $\chi^{2}$ a été utilisé pour les comparaisons statistiques de certains résultats.

\section{Résultats}

\section{Épidémiologie}

Durant la période d'étude, 60748 femmes ont été admises pour accouchement et 1704 pour avortement spontané. Pendant cette même période, nous avons recensé 24 cas de môle hydatiforme partielle (MHP) et 267 cas de môle hydatiforme complète (MHC). Les MHP représentent ainsi 0,04\% des accouchements normaux, soit $1 / 2500$.

La moyenne d'âge des patientes est de 26 ans. Une part numériquement importante des patientes admises pour une grossesse molaire partielle appartient aux tranches d'âge des 20 29 ans $(50,0 \%)$ et $30-39$ ans $(45,8 \%)$, mais aucune différence significative n'est observée pour le risque relatif en fonction de ces tranches d'âge (Tableau 1).

Le tableau 2 montre la répartition des patientes selon la parité; cette dernière ne semble pas être liée au développement d'une MHP.

À leur première admission dans notre structure, aucun antécédent de MHP n'a été retrouvé chez nos patientes ; par contre, quatre avaient déjà subi un avortement spontané non molaire.
Le groupage $\mathrm{ABO}$ était précisé chez touteslespatientes.Legroupesanguin $\mathrm{O}$ était le plus fréquent et représentait $54,1 \%$ des cas, suivi par le groupe A qui représentait $25,0 \%$ et le groupe B qui représentait 16,7\%. Ces différences ne sont pas significatives par rapport à la population générale (Tableau 3 ).

\section{Données cliniques}

Les différents motifs de consultation sont représentés dans le tableau 4 : les métrorragies représentaient de loin le motif le plus fréquent $(79,2 \%)$. Les algies pelviennes étaient retrouvées dans $62,5 \%$ des cas. Elles étaient en rapport avec la présence de kystes lutéiniques (Figure 1) ou l'expulsion de vésicules et d'embryon. Le syndrome toxique était présent chez $12,5 \%$ des patientes. Un syndrome thyrotoxicosique clinique était retrouvé chez une patiente. Ce syndrome comportait une tachycardie, une thermophobie, un tremblement des extrémités et un amaigrissement. La taille utérine était plus élevée par rapport à l'âge gestationnel chez $83,3 \%$ des cas de MHP.

\section{Examens paracliniques}

Excepté pour les patientes arrivant avec un tableau évident d'expulsion d'embryon et de vésicules ( $8,3 \%)$, les dosages de $\beta$-hCG réalisés dès l'admission pour les autres ont mis en évidence des taux élevés par rapport à l'âge gestationnel chez 91,7\% d'entre elles.

L'échographie pelvienne et/ou endovaginale a été pratiquée chez toutes les patientes. Elle permettait d'évoquer le diagnostic de MHP dans $87,5 \%$ des cas en objectivant des images dites en «flocons de neige $\gg$ ou en «nid d'abeilles » avec un embryon. Les kystes lutéiniques étaient retrouvés à l'échographie chez $12,5 \%$ des patientes.

La numération formule sanguine montrait une anémie chez $29,2 \%$ de nos patientes. 


\begin{tabular}{|c|c|c|c|c|c|c|}
\hline \multirow{2}{*}{$\begin{array}{l}\text { Âge maternel } \\
\text { (ans) }\end{array}$} & \multicolumn{2}{|c|}{ MHP } & \multicolumn{2}{|c|}{ Grossesses normales } & \multirow{2}{*}{$\begin{array}{l}\text { Incidence relative } \\
\text { des MHP (n/100) }\end{array}$} & \multirow{2}{*}{$\begin{array}{l}\text { Comparaison par rapport } \\
\text { aux accouchements }\end{array}$} \\
\hline & Nbre & $\%$ & Nbre & $\%$ & & \\
\hline$<20$ & 0 & 0,0 & 1269 & 2,1 & 0,0 & - \\
\hline $20-29$ & 12 & 50,0 & 33286 & 54,8 & 0,04 & NS \\
\hline $30-39$ & 11 & 45,8 & 25581 & 42,1 & 0,04 & NS \\
\hline$\geq 40$ & 1 & 4,2 & 612 & 1,0 & 0,16 & - \\
\hline Total & 24 & 100,0 & 60748 & 100,0 & 0,04 & - \\
\hline
\end{tabular}

$N S=$ différence non significative.

Tableau 2 Fréquence de la môle hydatiforme partielle (MHP) en fonction de la parité et son incidence parmi l'ensemble des échecs à la reproduction par avortement

\begin{tabular}{lcccc} 
Parité & & MHP & \multicolumn{2}{c}{ Avortements non molaires } \\
& Nbre & $\%$ & Nbre & $\%$ \\
0 & 4 & 16,7 & 307 & 18 \\
1 & 6 & 25,0 & 392 & 23 \\
2 & 8 & 33,3 & 324 & 19 \\
3 & 5 & 20,8 & 289 & 17 \\
$\geq 4$ & 1 & 4,2 & 392 & 23 \\
Total & 24 & 100 & 1704 & 100 \\
\hline
\end{tabular}

Une hyperthyroïdie biologique était retrouvée chez une patiente de notre série, avec un taux élevé des hormones thyroïdiennes et une TSH (thyroid stimulating hormone - thyréostimuline hypophysaire) basse.

\section{Traitement et résultats histologiques}

Un curetage aspiratif était réalisé chez $83,3 \%$ des patientes. Trois patientes avaient expulsé spontanément, et de ce fait, une intervention ne semblait pas requise. Une patiente était traitée par césarienne devant une hauteur utérine très élevée $(36 \mathrm{~cm})$ avec un utérus cicatriciel. Le diagnostic de MHP était ainsi confirmé à l'examen anatomopathologique pour toutes les patientes incluses dans cette série.

\section{Surveillance post-môlaire et évolution}

Elle consistait en une surveillance clinique (persistance des métrorragies, involution utérine), biologique (dosage hebdomadaire de $\beta$-hCG, puis mensuel jusqu'à la négativation) et radiologique (échographie pelvienne de contrôle). Toutes les patientes étaient suivies cliniquement et biologiquement. L'échographie pelvienne était demandée chez toutes les patientes tous les trois mois pendant un an.

À court terme (durant l'hospitalisation), l'évolution était marquée par la disparition des métrorragies avec une involution utérine dans tous les cas ; une régression des taux élevés de $\beta$-hCG était notée chez toutes les patientes.

À long terme (en extra-hospitalier), 95,8\% des cas de MHP évoluaient

\begin{tabular}{|c|c|c|c|c|c|}
\hline \multirow[t]{2}{*}{ Groupe sanguin } & \multicolumn{2}{|c|}{$\begin{array}{l}\text { Population générale de } 10000 \text { femmes } \\
\text { enceintes normales }\end{array}$} & \multicolumn{2}{|c|}{ MHP } & \multirow[t]{2}{*}{$p$} \\
\hline & Nbre & $\%$ & Nbre & $\%$ & \\
\hline $\mathrm{O}$ & 5000 & 50,0 & 13 & 54,1 & NS \\
\hline A & 2600 & 26,0 & 6 & 25,0 & NS \\
\hline B & 1711 & 17,1 & 4 & 16,7 & NS \\
\hline$A B$ & 689 & 6,9 & 1 & 4,2 & NS \\
\hline Total & 10000 & 100 & 24 & 100 & - \\
\hline
\end{tabular}

MHP : môle hydatiforme partielle.

NS = différence non significative. 
favorablement avec disparition des métrorragies, involution utérine et négativation du taux de $\beta$-hCG. Une patiente avait évolué vers une tumeur trophoblastique gestationnelle qui était traitée par monochimiothérapie. Elle avait bien réagi au traitement.

Le suivi de nos patientes révélait que 18 d'entre elles (75\%) ont eu une grossesse clinique après la guérison de la MHP. Parmi elles, 14 patientes avaient mené à terme une grossesse normale alors que deux avaient eu une fausse couche spontanée.

\section{Discussion}

Le nom de môle hydatiforme vient du mot grec «môle » qui signifie masse et du mot « hydatide » qui signifie sac hydrique. La MHP est un œuf humain pathologique comportant des villosités en transformation vésiculaire, môlaire, mais conservant une forme placentaire reconnaissable et une cavité amniotique avec un embryon, ou des traces d'embryon [1]. Dans le cas des MHC, la dégénérescence hydropique est totale etl'onn'observeaucunetraced'embryon, de cavité amniotique et même de vascularisation $[1,2,5,6]$. Les MHP représentent $85 \%$ des triploïdies [6], la triploïdie étant définie par un caryotype à 69 chromosomes $[1,2]$. C'est une situation relativement rare $[6,7]$.

Les MHP, ou môles embryonnées ou encore incomplètes, sont souvent amalgamées avec les môles hydatiformes qui sont complètes, diploïdes et sans embryon. L'ensemble des môles hydatiformes est une entité distincte de la triploïdie, tant anatomopathologiquement que sur le plan diagnostique et évolutif $[1,2]$.

Les MHP surviennent plus chez la femme jeune (pic de fréquence à 27 ans), ayant souvent des antécédents d'avortement spontané $[8,9]$. Dans notre série, cet antécédent était retrouvé dans $16,7 \%$ des cas. Cette fréquence élevée dans cette tranche

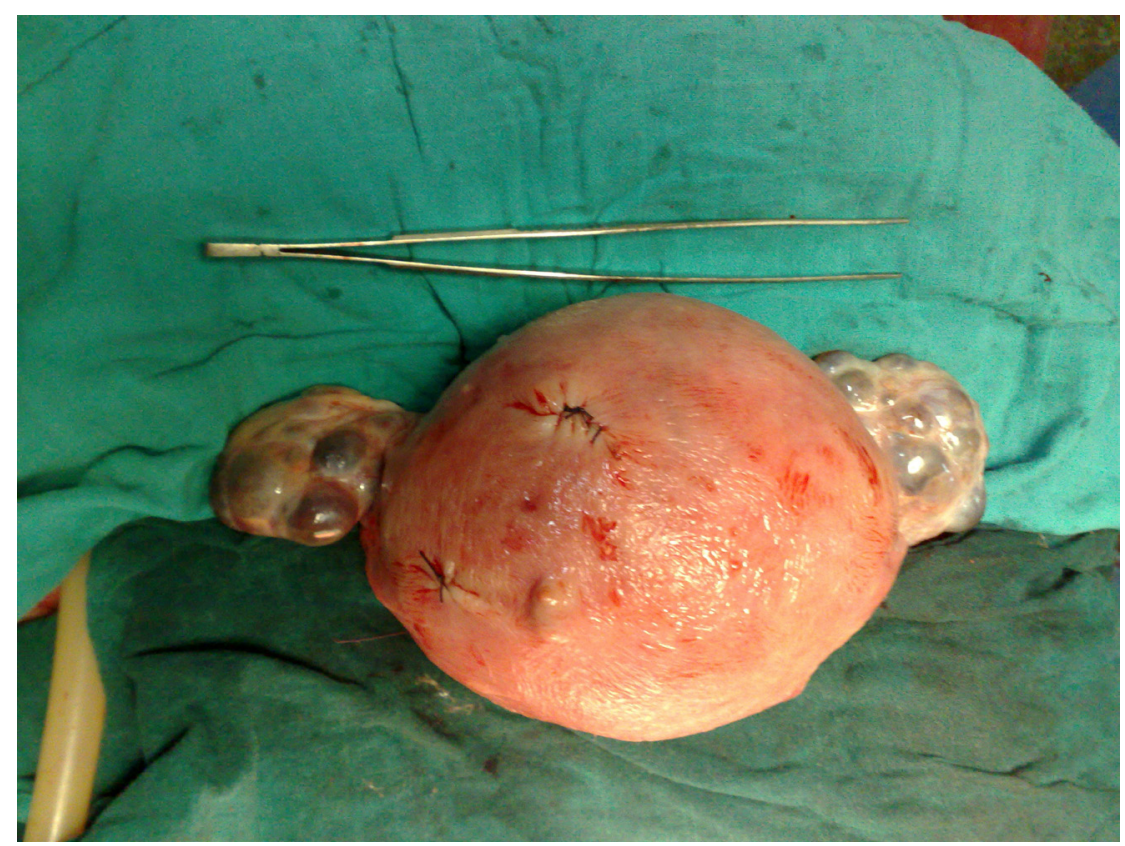

Figure 1 Photo per-opératoire montrant des kystes lutéiniques bilatéraux au cours d'une môle hydatiforme partielle d'âge est probablement expliquée par la fréquence de reproduction élevée à cet âge. D'ailleurs, dans notre série, la différence n'est pas significative entre la survenue de MHP et de grossesse normale. Le cas est différent pour la MHC, dont la survenue est très élevée dans les tranches d'âge extrêmes, comparée à la survenue de grossesses normales (différence significative) [2].

Dans les MHP, les facteurs de risque connus des môles hydatiformes complètes, notamment l'âge maternel supérieur à 40 ans, la susceptibilité familiale et le groupe sanguin $\mathrm{A}$, ne sont pas retrouvés $[1,2]$. En effet, l'âge est un facteur plus fortement associé à la MHC quà la
MHP [2]. Ceci conforte l'hypothèse selon laquelle le risque de fécondation d'un ovocyte anormal est plus élevé au commencement et à la fin de la vie reproductive d'une femme. Il est exceptionnel de décrire une MHP chez une femme âgée de plus de 40 ans $[9,10]$.

Les mécanismes des accidents chromosomiques conduisant à une MHP sont le plus souvent la fécondation d'un ovule haploïde par deux spermatozoïdes haploïdes, c'est la dispermie, ou bien par un spermatozoïde diploïde, c’est la diandrie [11]. La répartition des chromosomes sexuels donne trois types de triploïdies : 69 XXX, 69 XXY,

\begin{tabular}{|c|c|c|}
\hline \multicolumn{3}{|c|}{$\begin{array}{l}\text { Tableau } 4 \text { Fréquence des circonstances de diagnostic positif de la môle } \\
\text { hydatiforme partielle }\end{array}$} \\
\hline Circonstances de diagnostic & Nbre & $\%(n=24)$ \\
\hline Métrorragies & 19 & 79,2 \\
\hline Douleurs pelviennes & 15 & 62,5 \\
\hline Exagération des signes sympathiques & 3 & 12,5 \\
\hline Expulsion de vésicules & 2 & 8,3 \\
\hline Thyrotoxicose & 1 & 4,2 \\
\hline Hypertension artérielle & 1 & 4,2 \\
\hline
\end{tabular}


69 XYY. On estime à $70 \%$ les XXY. Les XYY seraient beaucoup plus rares car leur évolution au-delà de quatre semaines seraient impossible. Une nette prédominance de la formule XXX avec des durées de gestation plus longues en cas de triploïdie XXY est retrouvée [12]. La population triploïde a un temps de dédoublement augmenté, ce qui explique l'hypotrophie foetale, la fréquence des malformations et les taux persistants d'hCG par longévité cellulaire excessive du trophoblaste résiduel [13]. Les taux sanguins maternels très élevés de $\beta$-hCG et d'œstriol seraient expliqués par l'expression de gènes portés par le bras long du chromosome 19 paternel, impliqués dans la production hormonale [12].

Le diagnostic des MHP est souvent difficile en début de grossesse, les signes cliniques et paracliniques étant peu caractéristiques $[13,14]$. Seul un caryotype fotal permet un diagnostic prénatal de certitude [14]. La présentation clinique est habituellement celle d'une menace d'avortement ou de fausse couche spontanée du premier trimestre avec métrorragies [1]. On retrouve parfois des signes cliniques évoquant le caractère môlaire de la grossesse (vomissements très marqués, hauteur utérine excessive pour le terme et métrorragies d'abondance variable) $[14,15]$. Dans ce contexte, c'est l'échographie qui suspecte le diagnostic de môle partielle [7-9,16].

Au premier trimestre, les MHP sont souvent des avortements spontanés, avec des embryons normaux mais hypotrophes dans deux tiers des cas, ou malformés dans un tiers des cas [1,2]. Le placenta est épais, avec parfois des images vésiculaires évocatrices. Le diagnostic de môle embryonnée peut être suspecté dès la dixième semaine de gestation $[7,8]$.

Au deuxième trimestre, les MHP peuvent associer une néphropathie gravidique précoce, sous forme d'une néphropathie gravidique classique (association d'une protéinurie supérieure à $1 \mathrm{~g} / \mathrm{L}$, d'œdèmes des membres inférieurs et d'une hypertension artérielle) [15] ou d'une atteinte rénale pseudoglomérulonéphrétique, plus rare mais plus spécifique de la MHP [1]. Cette néphropathie serait due à une colonisation trophoblastique pathologique. Quelques cas de pré-éclampsie, voire de syndrome HELLP, sont décrits avec des MHP tardives [16]. Des métrorragies persistantes, des vomissements rares mais graves et incoercibles, et un excès de volume utérin, qui est dû le plus souvent à l'évolution d'un hydramnios aigu (la hauteur utérine peut être normale ou faible en cas d'hypotrophie ou de mort foetale), sont retrouvés dans ces cas [16,17]. Une hypertrophie thyroidienne a été rapportée [17]. Les kystes ovariens lutéiniques sont rares [1,2] ; ils étaient retrouvés dans un cas de notre série.

Les taux excessifs d'hormone gonadotrophine chorionique reflètent l'importance de l'hyperplasie trophoblastique [1]. Le retour à la normale s'effectue en six semaines en moyenne. Le délai de régression est corrélé à l'importance des chiffres initiaux [1,2]. L'alpha-fœto-protéine sérique est élevée même en l'absence de malformation foetale associée.

L'échographie est extrêmement sensible et spécifique avec des critères diagnostiques bien définis : le placenta est volumineux et hétérogène, une partie du trophoblaste est d'aspect normal tandis que l'autre comprend un tissu d'échogénicité différente,comblant la majeure partie de la cavité et d'aspect en flocons de neige. On y retrouve de nombreuses cavités kystiques caractéristiques $[5,7,8]$. La présence d'un sac gestationnel est obligatoire pour définir une môle embryonnée. Le fœus présente un retard de croissance intra-utérin et la grossesse est le plus souvent non évolutive. Deux critères échographiques, lorsqu'ils sont présents, distinguent une MHP dans $87 \%$ des cas $[7,8]$ : d'une part, le rapport mesure transverse sur mesure antéropostérieure du sac gestationnel, qui est supérieur à 1,5 dans les môles; d'autre part, les modifications morphologiques du sac gestationnel qui est plus irrégulier et l'échogénicité de la réaction déciduale qui est plus importante au niveau de l'interface placenta-myomètre. La taille disproportionnée de la vésicule vitelline oriente vers une grossesse molaire partielle [7].

Au second trimestre, un retard de croissance intra-utérin (RCIU) sévère et des anomalies morphologiques sont visualisées dans $93 \%$ des cas $[1,7]$. L'aspecten « gruyère » etl'élargissement du placenta (plus de $4 \mathrm{~cm}$ entre 18 et 22 semaines d'aménorrhée) conforte le diagnostic [17]. Cet aspect peut poser le problème du diagnostic différentiel avec dégénérescence hydropique bénigne des villosités placentaires. Dans cette situation, les taux d'hCG sont normaux et le caryotype fotal, s'il est réalisé, est normal [1].

L'échographie ne dispense pas de l'histologie. Macroscopiquement, le matériel de la MHP est généralement moins abondant que dans la MHC avec un mélange de villosités môlaires et non môlaires. Le foetus comporte des anomalies, un hygroma cervical [18]. Microscopiquement, contrairement à la MHC, il existe un mélange de villosités môlaires et non môlaires. Le revêtement villositaire forme des invaginations et kystes à double revêtement cytotrophoblastique et syncytiotrophoblastique. Une cavité amniotique et des débris fœtaux sont fréquemment retrouvés [1]. Au caryotype, la MHP est généralement triploïde (69 XXX, 69 XXY ou 69 XYY) [1,2]. L'étude cytogénétique des MHP montre une triplöidie diandrique : deux lots de chromosomes d'origine paternelle et un lot d'origine maternelle [10]. 
Le diagnostic différentiel de la MHP se pose avec la triploïdie non molaire. Dans cette dernière, deux lots de chromosomes maternels sont retrouvés (triploïdie digynique) etl'arrêt de l'embryogenèse est précoce. Il se pose aussi avec la rétention intra-utérine prolongéeavecplacentahydropique [1]. L'étude immunohistochimique à l'aide d'anticorps anti-phosphatase alcaline placentaire montre de petits foyers marqués dans le placenta de décès in utero alors que ce marquage est plus diffus dans la môle partielle. Dans ce cas, le caryotype est normal et les taux de $\beta$-hCG sont normaux. Les grossesses môlaires partielles peuvent aussi prêter à confusion avec une môle hydatiforme complète associée à une grossesse normale. Dans ce cas, on retrouve un fotus et un placenta normaux et une môle hydatiforme complète. Les taux de $\beta$-hCG sont très élevés et l'alpha-foetoprotéine est normale [19].

\section{Surveillance post- thérapeutique}

Une surveillance clinique, échographique et biologique s'impose après évacuation d'une grossesse môlaire afin de détecter précocement toute évolution vers la tumeur trophoblastique gestationnelle [2022]. Le risque de tumeur trophoblastique gestationnelle (TTG) après une MHP est de 2 à $4 \%$. Ce risque augmente avec la durée de la grossesse [22-24] et la rapidité de l'intervention humaine. Dans notre série, une patiente avait évolué vers la TTG, qui avait bien répondu sous monochimiothérapie. Les autres cas avaient évolué favorablement après l'évacuation molaire. Nos résultats rejoignent ceux de la littérature qui rapportent une évolution favorable dans plus de $96 \%$ des MHP $[1,2]$. Le délai entre l'évacuation utérine et le passage à la TTG est court (entre 5 et 18 semaines) par rapport aux MHC. Dans tous les cas rapportés, la rémission a été obtenue par une chimiothérapie $[1,22]$.

La majorité des auteurs suggère de suivre la décroissance du taux de $\beta$-hCG plasmatique jusqu'à ce qu'il soit indétectable durant trois à six mois suivant l'évacuation utérine [19].

Une grossesse après l'évacuation môlaire se déroule souvent sans anomalie, mais elle risque de retarder le diagnostic d'une éventuelle tumeur trophoblastique gestationnelle postmôlaire en obscurcissant le monitoring du taux de $\beta$-hCG $[1,2]$. Ainsi, une contraception orale doit être démarrée dès l'évacuation et se maintenir pendant six mois jusquà un an après la normalisation du dosage de $\beta$-hCG [23].

La récidive d'une MHP est rare. Le risque de récidive est de $0,6 \%$ à $2 \%$ selon les études $[1,2]$. Sur une série exhaustive de cas, Sebire et al. [24] rapportent un risque de récidive, après un antécédent de MHP, de $1,7 \%(25 / 1512)$. Ce risque est de $1,9 \%$ après une MHC (27/1417). Le risque de récidive est beaucoup plus élevé chez les patientes ayant une mutation $\mathrm{du}$ gène NLRP7 [25].

Le type de môle récidivant est le plus souvent identique au cas antécédent. Sebire et al. montrent que $69 \%$ des récidives sont des MHP et $23 \%$ sont des MHC après un antécédent de MHP, alors que $77 \%$ des récidives sont des MHC et $23 \%$ sont des MHP après un antécédent de MHC [24]. Des cas de récidive à répétition de MHP ont été rapportés. Slim et al. ont montré que certains ovocytes d'une patiente avec mutation du gène NLRP7 ne sont pas en mesure d'empêcher la fécondation polyspermique. Cette mutation expliquerait les récidives de MHP, de même que de MHC, ainsi que l'association de plusieurs types de môles hydatiformes. La mise en évidence de ces gènes permettrait de caractériser la complexité génétique des tissus molaires et d'éviter le gaspillage en matière de reproduction chez certaines patientes [25].

\section{Fertilité après môle hydatiforme partielle}

Une grossesse après l'évacuation môlaire est possible et se déroule souvent sans anomalie pourvu qu'elle soit bien suivie. En revanche, une nouvelle grossesse précoce risque de retarder le diagnostic d'une éventuelle tumeur trophoblastique gestationnelle post-môlaire en obscurcissant le monitoring du taux de $\beta$-hCG [1]. Un délai de six mois à un an, assorti d'une contraception dont le choix doit être discuté avec la patiente, semble la norme à respecter en cas de MHP. La contraception orale n'augmente pas l'incidence de tumeur gestationnelle trophoblastique post-môlaire. Elle permet de détecter un éventuel regain prolifératif de la môle et de le traiter précocement. Elle doit être démarrée dès l'évacuation et se maintenir de six mois à un an après la normalisation du dosage de $\beta$-hCG[23]. Il n'y a pas d'augmentation de fréquence d'avortement spontané, ni de prématurité, ni de malformations congénitales par rapport à la population générale $[1,2,23]$. Parmi nos patientes qui ont eu une grossesse après leur première prise en charge dans notre service, aucun avortement ni aucune malformation n'étaient notés. En cas de grossesse, une échographie obstétricale doit être effectué le plus tôt possible ; après l'accouchement, le placenta doit être envoyé à l'anatomo-pathologiste et un dosage de $\beta$-hCG doit être réalisé à six semaines du post-partum $[1,2]$.

\section{Conclusion}

Les MHP constituent un accident génétique relativement rare. Elles présentent des caractéristiques histopathologiques et cytogénétiques ainsi qu'une évolution relativement distinctes des MHC. Des facteurs, notamment l'âge maternel, la parité, 
le groupe sanguin, le rôle des facteurs exogènes, ne se trouvent pas associés à la MHP, contrairement à la MHC. Des évolutions au-delà du premier trimestre sont rares mais possibles dans la MHP ; elles se présentent sous forme de môle embryonnée avec un retard de croissance fœital précoce et majeur. L'échographie tient une place essentielle dans le diagnostic, qui est fortement suspecté sur les critères cliniques et échographiques, et confirmé par l'obtention rapide d'un caryotype foetal par amniocentèse.
Le pronostic maternel est rarement menacé par l'évolution vers une môle invasive ou une tumeur trophoblastique gestationnelle. Les récidives sont exceptionnelles. Le pronostic fotal est caractéristique (malformations). La révélation de l'origine paternelle par étude des caryotypes paternels et recherche des marqueurs génétiques ne modifie pas le pronostic, et est donc sans intérêt pratique. Ainsi, accompagnées d'une surveillance et de soins particuliers, les MHP ont souvent une évolution favorable et n'altèrent pas, a priori, l'avenir obstétrical de la femme, ni du nouveau-né.

La création d'un centre de référence des maladies trophoblastiques gestationnelles dans notre pays s'avère nécessaire, non seulement pour améliorer le diagnostic et la prise en charge des patientes mais aussi pour élucider certains phénomènes qui génèrent cette maladie.

\section{Références}

1. Golfier F et al. Évaluation de la prise en charge des tumeurs trophoblastiques gestationnelles enregistrées au Centre de référence des maladies trophoblastiques de Lyon de 1990 à 2005 [Evaluation of treatment relating to gestational trophoblastic tumor registered to the French Trophoblastic Disease Reference Center (TDRC) in Lyon from 1999 to 2005]. Gynécologie, Obstétrique \& Fertilité, 2007, 35(3):205-215.

2. Boufettal $\mathrm{H}$ et al. Les môles hydatiformes complètes au Maroc : étude épidémiologique et clinique [Complete hydatiform mole in Morocco: epidemiological and clinical study]. Journal de gynécologie, obstétrique et biologie de la reproduction, 2011, 40(5):419-429.

3. Jacobs PA et al. Human triploidy: relationship between parental origin of the additional haploid complement and development of partial hydatidiform mole. Annals of Human Genetics, 1982, 46:223-231.

4. Lawler SD, Fisher RA, Dent J. A prospective genetic study of complete and partial hydatidiform moles. American Journal of Obstetrics and Gynecology, 1991, 164:1270-1277.

5. Flici O et al. Môle embryonnée : apport de l'échographie. À propos de neuf cas [Embryonal mole: value of echography. Report of 9 cases]. Journal de gynécologie, obstétrique et biologie de la reproduction, 1983, 12:167-176.

6. Jacobs PA et al. Complete and partial hydatidiform mole in Hawaii: cytogenetics, morphology and epidemiology. BJOG, 1982, 89:258-266.

7. Fine $\mathrm{C}$ et al. Sonographic diagnosis of partial hydatidiform mole. Obstetrics and Gynecology, 1989, 73:414-418.

8. Jauniaux E. Ultrasound diagnosis and follow-up of gestational trophoblastic disease. Ultrasound in Obstetrics \& Gynecology, 1998, 11:367-377.

9. Genest DR. Partial hydatidiform mole: clinicopathological features, differential diagnosis, ploidy and molecular studies, and gold standards for diagnosis. International Journal of Gynecological Pathology, 2001, 20:315-322.

10. Folkins A et al. Utility of chromosomal chromogenic in situ hybridization as an alternative to flow cytometry and cytogenetics in the diagnosis of early partial hydatidiform moles: a validation study. Journal of Reproductive Medicine, 2010, 55:275-278.

11. Sebire NJ et al. Shortened duration of human chorionic gonadotrophin surveillance following complete or partial hydatidiform mole: evidence for revised protocol of a UK regional trophoblastic disease unit. BJOG, 2007, 114:760-762.

12. Lavie I et al. Duration of human chorionic gonadotropin surveillance for partial hydatidiform moles. American Journal of Obstetrics and Gynecology, 2005, 192:1362-1364.
13. Berkowitz RS et al. Case-control study of risk factors for partial molar pregnancy. American Journal of Obstetrics and Gynecology, 1995, 173:788-794.

14. Hayati AR, Tan GC. Clinicopathologic and immunohistochemical differences in complete and partial hydatidiform moles in a multiracial Malaysian population. International Journal of Gynecological Pathology, 2005, 24:277285.

15. Berkowitz RS et al. Case-control study of risk factors for partial molar pregnancy. American Journal of Obstetrics and Gynecology, 1995, 173:788-794.

16. Falkert A, Yildiz A, Seelbach-Goebel B. Partial mole with fetal triploidy as a cause for imminent HELLP-syndrome at 16 weeks of gestation. Archives of Gynecology and Obstetrics, 2009, 279:423-425.

17. Chiniwala NU et al. Thyroid storm caused by a partial hydatidiform mole. Thyroid, 2008, 18:479-481.

18. Medeiros F et al. Intraplacental choriocarcinoma arising in a second trimester placenta with partial hydatidiform mole. International Journal of Gynecological Pathology, 2008, 27:247-251.

19. Matsui $\mathrm{H}$ et al. Subsequent pregnancy outcome in patients with spontaneous resolution of HCG after evacuation of hydatidiform mole: comparison between complete and partial mole. Human Reproduction (Oxford, England), 2001, $16: 1274-1277$.

20. Rice LW et al. Persistent gestational trophoblastic tumor after partial hydatidiform mole. Gynecologic Oncology, 1990, 36:358-362.

21. Feltmate $\mathrm{CM}$ et al. Clinical characteristics of persistent gestational trophoblastic neoplasia after partial hydatidiform molar pregnancy. Journal of Reproductive Medicine, 2006, 51:902-906.

22. Hancock BW, Nazir K, Everard JE. Persistent gestational trophoblastic neoplasia after partial hydatidiform mole incidence and outcome. Journal of Reproductive Medicine, 2006, 51:764-766.

23. Costa H, Doyle P. Influence of oral contraceptives in the development of post-molar trophoblastic neoplasia-A systemic review. Gynecologic Oncology, 2009, 100:579-585.

24. Sebire NJ et al. Risk of recurrent hydatidiform mole and subsequent pregnancy outcome following complete or partial hydatidiform molar pregnancy. BJOG, 2003, 110:22-26.

25. Slim R et al. Recurrent triploid and dispermic conceptions in patients with NLRP7 mutations. Placenta, 2011, 32:409-412. 